\title{
Laser-Inscribed Stress-Induced Birefringence of Sapphire
}

\author{
Hua Fan ${ }^{1}$, Meguya Ryu ${ }^{2}$, Reo Honda ${ }^{2}$, Junko Morikawa ${ }^{2,3}$, Zhen-Ze Li ${ }^{1}$, Lei Wang ${ }^{1}$ (D, \\ Jovan Maksimovic ${ }^{4,5}$, Saulius Juodkazis ${ }^{3,4,5, *}$, , Qi-Dai Chen ${ }^{1, *}$ and Hong-Bo Sun ${ }^{6}$ \\ 1 State Key Laboratory of Integrated Optoeletronics, College of Electronic Science and Engineering, \\ Jilin University, Changchun 130012, China; fanhua17@mails.jlu.edu.cn (H.F.); zhenze_lee@163.com (Z.-Z.L.); \\ leiwang1987@jlu.edu.cn (L.W.) \\ 2 School of Materials and Chemical Technology, Tokyo Institute of Technology, Meguro-ku, \\ Tokyo 152-8550, Japan; ryu.m.ab@m.titech.ac.jp (M.R.); leo_0520@icloud.com (R.H.); \\ morikawa.j.aa@m.titech.ac.jp (J.M.) \\ 3 Tokyo Tech World Research Hub Initiative (WRHI), School of Materials and Chemical Technology, \\ Tokyo Institute of Technology, 2-12-1, Ookayama, Meguro-ku, Tokyo 152-8550, Japan \\ 4 Centre for Micro-Photonics, Faculty of Science, Engineering and Technology, \\ Swinburne University of Technology, Hawthorn, VIC 3122, Australia; jmaksimovic@swin.edu.au \\ 5 Melbourne Centre for Nanofabrication, ANFF, 151 Wellington Road, Clayton, VIC 3168, Australia \\ 6 State Key Laboratory of Precision Measurement Technology and Instruments, \\ Department of Precision Instrument, Tsinghua University, Beijing 100084, China; hbsun@jlu.edu.cn \\ * Correspondence: saulius.juodkazis@gmail.com (S.J.); chenqd@jlu.edu.cn (Q.-D.C.)
}

Received: 19 August 2019; Accepted: 26 September 2019 ; Published: 3 October 2019

\begin{abstract}
Birefringence of $3 \times 10^{-3}$ is demonstrated inside cross-sectional regions of $100 \mu \mathrm{m}$, inscribed by axially stretched Bessel-beam-like fs-laser pulses along the c-axis inside sapphire. A high birefringence and retardance of $\lambda / 4$ at mid-visible spectral range (green) can be achieved using stretched beams with axial extension of 30-40 $\mu \mathrm{m}$. Chosen conditions of laser-writing ensure that there are no formations of self-organized nano-gratings. This method can be adopted for creation of polarization optical elements and fabrication of spatially varying birefringent patterns for optical vortex generation.
\end{abstract}

Keywords: femtosecond laser; birefringence; stress; sapphire

\section{Introduction}

Three-dimensional (3D) structuring of materials with a high refractive index at sub-wavelength resolution has promise to advance the field of photonic crystals $(\mathrm{PhC})$ and the integration of $\mathrm{PhC}$ into photonic chips [1-11]. Femtosecond laser micro/nano-fabrication as the no contact method can directly pattern sub-wavelength ripples [12,13]). A 3D nonlinear $\mathrm{PhC}$ has been successfully fabricated inside lithium niobate using femtosecond laser [14]. However, this is still a challenging task [15] to deliver a close to diffraction-limited focusing at arbitrary depths required for the 3D patterning when using Gaussian-like laser pulses [16]. Compensation of spherical aberrations can be successfully achieved for laser-writing in high refractive index materials and large depths [17]. In this study, we enhance (instead of compensating [17]) the spherical aberration by tailoring axial light intensity to be stretched along the propagation direction and to form a Bessel-beam-like axial intensity profile. Similar techniques are used for 3D patterning inside high refractive index materials $[5,6,8,18]$. We use stretched pulses to control 3D structuring and dielectric permittivity change over tens-of-micrometers along the entire typical length of fs-laser pulses of 100-300 fs. 
Permittivity $\varepsilon=n^{2}$ changes between the laser-inscribed region $\varepsilon_{1}$ (refractive index $n_{1}$ ) with width $t_{1}$ separated with a host material of permittivity $\varepsilon_{2}$ and width $t_{2}$, creates an artificial uniaxial form-birefringent structure with $\varepsilon_{e}-\varepsilon_{o}>0$, where $e, o$ denotes extraordinary and ordinary beams polarized $\|$ and $\perp$ to the optical axis $O A$, respectively. Controlling the width of $t_{1}$, its period $t_{1}+t_{2}$ and depth $d$ (along the light propagation) are essential parameters to an engineer's optical elements for polarization control. Stress-induced birefringence is a well-used phenomenon to create artificial birefringent materials and used as a method for material inspection and characterization $[19,20]$. Generally, the inverse permittivity tensor is $\Delta\left(1 / \varepsilon_{i j}\right)=P_{i j k l} \partial_{k} u_{l}$, where $P_{i j k l}$ is the fourth-rank photoelasticity tensor, $\partial_{k} u_{l}$ is the gradient of the displacement from equilibrium with $u_{l}$ being the linear displacement from equilibrium and $\partial_{k}$ denotes differentiation with respect to the Cartesian coordinates. Birefringence induced by stress and expanding beyond laser-structured regions is promising for polarization micro-optics, especially when the laser modified regions are not used for optical functions due to considerable light scattering losses.

In any type of material, the modification of adjacent regions with resolution smaller than the wavelength can be used to change the effective refractive index which for extraordinary $n_{e}$ (along the optical axis) and ordinary $n_{o}$ indices depend on the volume fraction $f=t / \Lambda$ [21]:

$$
n_{e}=\sqrt{\frac{n_{1}^{2} n_{2}^{2}}{f n_{2}^{2}+(1-f) n_{1}^{2}}} ; \quad n_{o}=\sqrt{f n_{1}^{2}+(1-f) n_{2}^{2}}
$$

where $n_{1}$ and $n_{2}$ are the indices of the host and laser-inscribed regions, respectively. If modification of a silica host $n_{1}=1.40$ becomes $n_{1}=1.45$, the form birefringence would only reach maximum of $\Delta n=n_{e}-n_{o}=-8.8 \times 10^{-4}$ when $f=0.5$, i.e., $t=\Lambda / 2$. Hence, for a considerable phase delay required for $\lambda / 4$ or $\lambda / 2$ polarization optics, a tens-of- $\mu$ m-long axial modification $d$ would be required for engineering the retardance $\Delta n \times d$. Moreover, a well-controlled laser inscription method developed here is required to reach the optimal conditions of $f=0.5$ for the shortest modification $d$ at the chosen wavelength of operation (the strongest birefringence $\Delta n$ ). Such flexibility is currently not available for fabrication of micro-optical elements.

Here, we show that single nanolines array with controllable separation inscribed in crystalline sapphire (along $c$-axis) can reach retardance of $\lambda / 4(\pi / 2$ in phase) for visible wavelengths using a simple approach to generate femtosecond pulses with an axially extended Bessel-like intensity distribution using a spatial light modulator (SLM).

\section{Experimental}

C-cut sapphire samples of $0.5 \mathrm{~mm}$ thickness were used for laser inscription. Sapphire has one of the highest Young moduluses of $Y=400 \mathrm{GPa}$ and can withstand high pressures strongly localized inside the crystal, as our earlier studies as shown in our earlier studies [22]. The stress-induced birefringence inside the laser-structured region patterned with single pulse irradiation reached $\Delta n \approx 1 \times 10^{-3}$ and the pressure was estimated to reach 1.3 GPa. At $\sim 2 \mathrm{GPa}$, micro-cracks were developed when Gaussian fs-laser pulses were used in c-cut sapphire [22].

In this study, a fs-laser beam at second harmonic $\lambda=515 \mathrm{~nm}$ wavelength and pulse duration of $t_{p}=280 \mathrm{fs}$ (Pharos, Light Conversion) was reflected from a phase mask designed on a spatial light modulator (SLM) and directed onto a tight focusing objective lens for laser structuring. Typical pulse energy used for stress-induced birefringent gratings was $E_{p}=574 \mathrm{~nJ}$ (at focus) to inscribe single modification lines inside sapphire at laser repetition rate of $10 \mathrm{kHz}$ at a beam scanning speed of $0.1 \mathrm{~mm} / \mathrm{s}$ (if not specified otherwise). The focal diameter was $\sim 465 \mathrm{~nm}$ which can be calculated approximately using the formula $d=1.22 \lambda / N A$. The phase mask pattern on the SLM was selected to create close-to-linear intensity distribution along the propagation direction and was characterized by a stretch factor $f_{s t}$. For the $f_{s t}=0$ the spherical aberration was compensated at the position of the Gaussian beam, while the largest value of $f_{s t}=12$ was at the maximum stretch to 
obtain a linear intensity distribution over the entire pulse length $c t_{p} / n$, here $t_{p}$ is the pulse duration. The inscribed structure was approximately 10-15 $\mu \mathrm{m}$ below the sample surface. A laser-structured sapphire sample was cleaved to expose the structured regions and was etched in $20 \% \mathrm{HF}$ for $60 \mathrm{~min}$ before SEM observation.

\section{Results and Discussion}

By rational choice of repetition rate and scanning speed, it was possible to create a single line without movement of the sample (or beam). By scanning the beams linearly modified regions, lengths of tens-of-micrometers were recorded. For the most axially stretched intensity distribution, single lines were recorded without usual formation of nanogratings [4,23]. First, we present structural characterisation of inscribed modified lines at a wider range of parameters and subsequently present results of optical characterisation of patterns which can deliver a $\lambda / 4$ waveplate performance.

\subsection{Direct Write of Nano-Planes}

Inscription of long axial modifications in a crystalline sapphire were made by focusing onto a c-plane sample. Typical results of laser inscription are summarized in Figure 1. For same stretch factors $f_{s t}=9$, different pulse frequency was used to study the evolutionary process of the femtosecond laser irradiated area (Figure 1b,c). The property of the irradiated (modified) area has been demonstrated in our previous work, which present the phase transition of the modified area from crystalline to amorphous in sapphire after irradiated by femtosecond laser [12]. 20\% HF solution was employed due to the very high contrast of etching between crystalline sapphire and laser amorphized regions ( $\sim 10^{4}$ etching selectivity) such as those seen previously when using silica and boro-silicate glasses [24-28]. Etching up to $0.8 \mathrm{~mm}$ into the depth of sapphire sample (along $z$-axis; inset in Figure 1a) was observed when continuous inscriptions of strongly modified regions were formed at large frequency.

(a) $f_{s t}=9 f: 1 K$

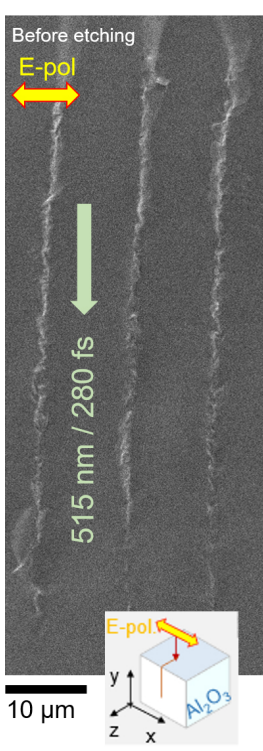

(b) $f_{s t}=9 f: 1 K$

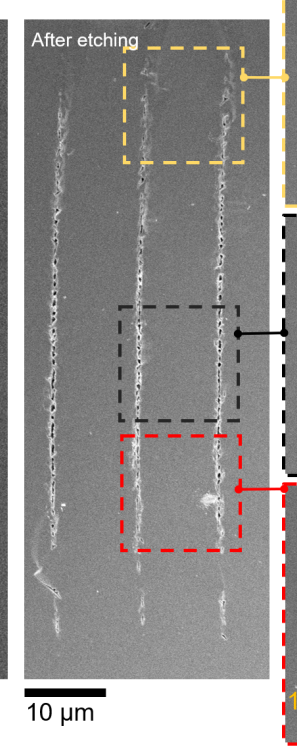

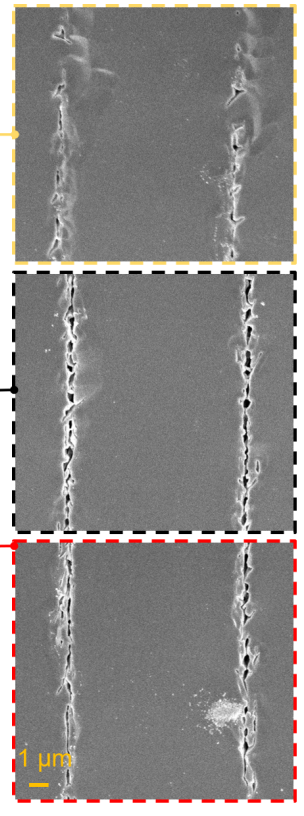

(c) $f_{s t}=9 f: 10 \mathrm{~K}$

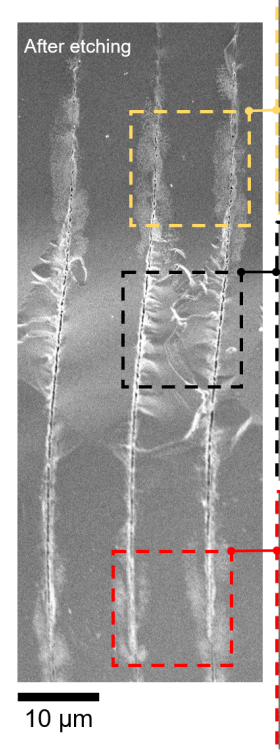

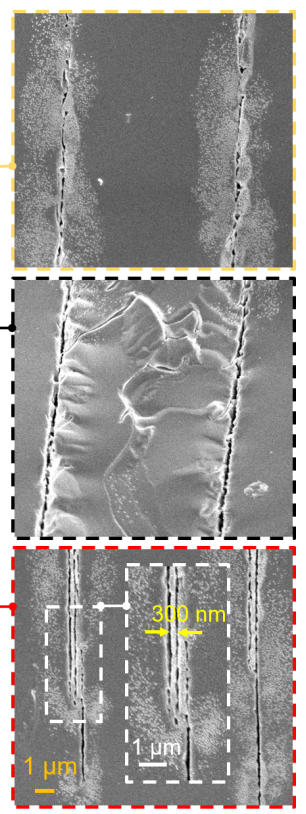

Figure 1. Inscription of sapphire with Bessel-beam at different stretch factors $f_{s t}$ ( 0 corresponds to the spherical aberration compensation while the largest value of 12 was for the maximum stretch to obtain a linear intensity distribution over the entire pulse length $c t_{p} / n$, here $t_{p}$ is the pulse duration). SEM side-view images of as fabricated sample after breaking it on a xy-plane (see the inset) (a) and after wet etching in HF $20 \%$ vol. for $60 \mathrm{~min}(\mathbf{b}, \mathbf{c})$ with same single pulse $E_{p}=847 \mathrm{~nJ}$ at different pulse frequency: $1 \mathrm{kHz}(\mathbf{a}, \mathbf{b}), 10 \mathrm{kHz}(\mathbf{c})$; the beam was scanned at $v_{s}=0.5 \mathrm{~mm} / \mathrm{s}$ along $z$-axis. Some lines do not appear straight in SEM images due to the uneven surface of the cleaved region. 
For smaller pulse number and the same $f_{\text {st }}$, the individual laser damaged nano-regions, which were not initially interconnected into a single line, were observed connected after wet etching. With an increasing number of pulses (larger repetition rate or slow scanning), those single damage regions formed a line, which was further revealed after etching.

The longest inscription of modifications of up to $60 \mu \mathrm{m}$ along the propagation direction (on $y$-axis) were inscribed with $\sim 850 \mathrm{~nJ} /$ pulse energy at $f_{s t}=9, f=10 \mathrm{kHz}$ repetition rate and scanning speed of $v_{s}=0.5 \mathrm{~mm} / \mathrm{s}$ along $z$-axis (Figure 1a). At these conditions approximately $n=d / v_{s} / f \approx 46$ pulses were accumulated over the diameter of the focal spot $(\sim 465 \mathrm{~nm})$. Due to the large pulse energy, gratings with period of $\sim 300 \mathrm{~nm}$ were formed in the modified region as revealed after wet etching (inset in Figure 1c). The incident and scattered/reflected light from the strongly excited region interfere and form nanograting patterns as was described in ref. [29]. Near-sub-wavelength structures with a period of $\lambda / n=515 \mathrm{~nm} / 1.45=355 \mathrm{~nm}$ were revealed in the strongly absorbing regions where the pulse-induced permittivity became metal-like.

As shown in Figure 2, an array of uniform non-period nanolines with $1 \mu \mathrm{m}$ interspace was inscribed by stretched fs-laser pulse (energy $574 \mathrm{~nJ}$, scanning speed $0.1 \mathrm{~mm} / \mathrm{s}$ ). The width and length of the single nanoline after etching in HF solution were $50 \mu \mathrm{m}$ and $\sim 170 \mathrm{~nm}$, respectively. The aspect ratio of the lines can reach 250 , which pave the way to inscribe a strong and tunable birefringence and retardance in sapphire.

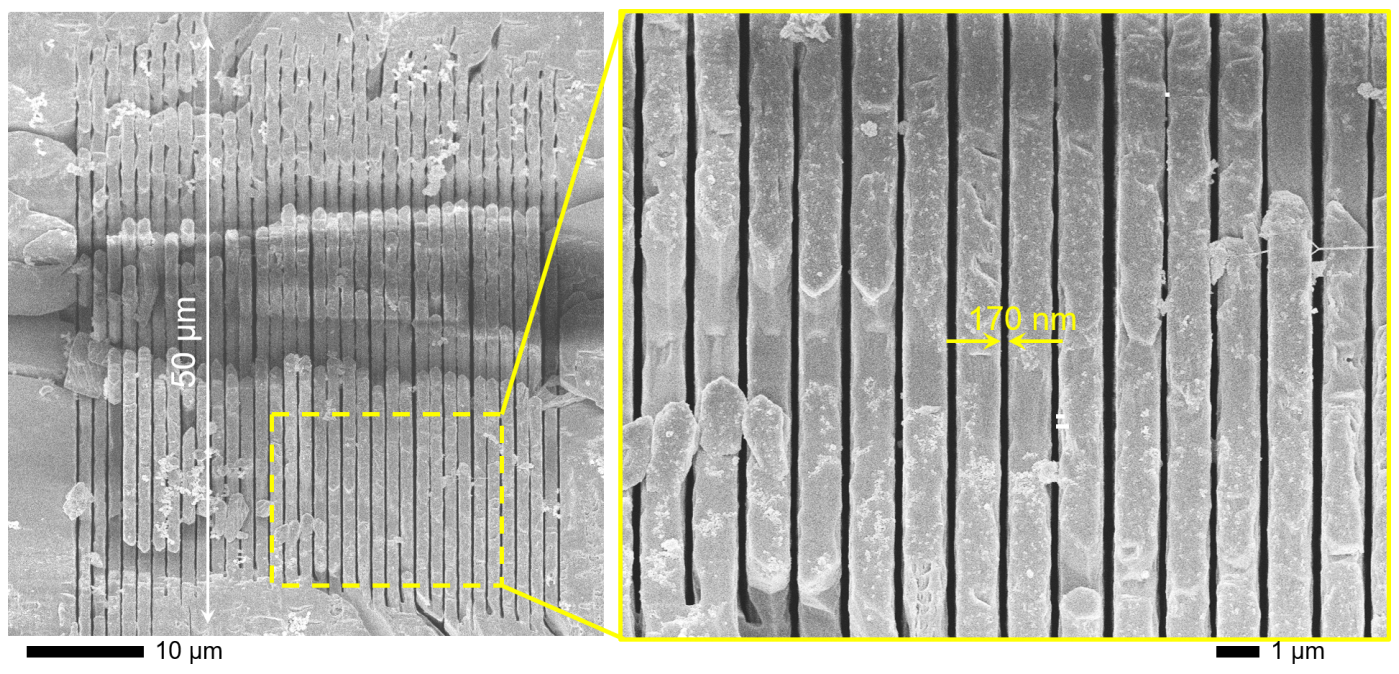

Figure 2. SEM images of the highest resolution single-line pattern inscribed in sapphire at the largest stretch factor $f_{s t}=12$; conditions as in Figure 1a. The sample was cleaved (image plane) at some depth along the inscribed pattern and wet-etched for the SEM observation. The single line was inscribed by Bessel-like beam scanning without formation of periodicity nano-gratings $\left(f_{s t} \leq 9\right)$ nor transitional irregular pattern at low frequency and high pulse energy (Figure 1b). Wet-etched patterns reached aspect ratio $50 / 0.2=250$.

The use of an even distribution of pulse energy along the propagation axis can be applied in generation of high pressure and temperature phases of materials due to better energy delivery via resonant absorption [30]. The proposed phase control using SLM can, in principle, be adopted for experiments exploring a temporal evolution of fs-laser pulse-induced micro-explosions using femtosecond X-ray pulses of a free electron laser (FEL) for probing, while coaxially propagating Bessel-like beams [31] can be used for optically triggered micro-explosions. The current study confirms formation of an amorphous phase of sapphire which is typical in conditions of high pressure [32].

\subsection{Engineering of Birefringence}

Optical characterization of laser-inscribed gratings are shown in Figure 3. Gratings with $\Lambda=10 \mu \mathrm{m}$ period were inscribed with duty cycle of 0.5 , i.e., $10 \mu \mathrm{m}$ were inscribed with a separation 
of $\Delta x$ ranging from $170 \mathrm{~nm}$ to $500 \mathrm{~nm}$ between two single nanolines. The footprint of the gratings were $100 \times 100 \mu \mathrm{m}^{2}$, an acceptable size for many applications in the field of micro-optical elements.
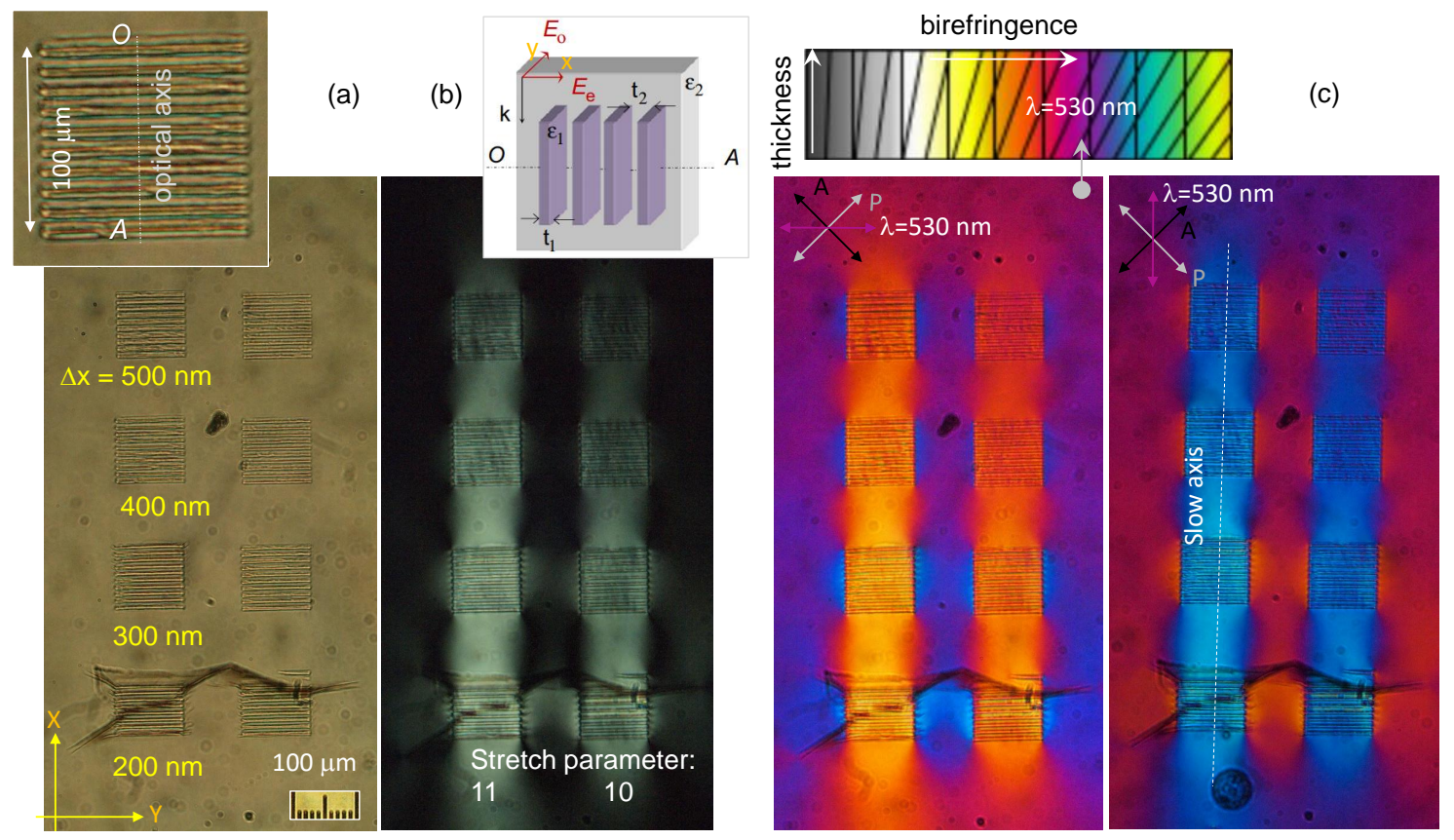

Figure 3. Optical (a), cross-polarized (b) and color-shifted birefringence with $\lambda=530 \mathrm{~nm}$ waveplate at two orientations (c) images of the sample between polarizer $(\mathrm{P})$ and crossed-oriented analyzer (A). The inset in (a) shows the pattern of $10 \mu \mathrm{m}$ inscribed regions with nano-planes with separation of $\Delta x$. The form-birefringent pattern of negative uniaxial crystal $\Delta x=t_{1}+t_{2}$ and the orientation of ordinary and extraordinary fields $E_{o, e} ; O A$ is the optical axis (inset in (b)). The stretch parameter of 11 corresponds to $\sim 40 \mu \mathrm{m}$ axial extent of the laser-inscribed region, 10 corresponds to $\sim 30 \mu \mathrm{m}$. The Michel-Levy birefrincence color chart is shown in the inset of (c).

Single lines without the formation of self-organized nano-gratings were inscribed by stretching the incoming fs-laser pulses. The stretch factors of 10 and 11 corresponded to a single line (a plane under scanning) inscription for $30 \mu \mathrm{m}$ and $40 \mu \mathrm{m}$, respectively. Inspection of the laser-inscribed regions with scanning electron microscopy (SEM) revealed the width of the structurally modified lines corresponding to $\sim 170 \mathrm{~nm}$ (Figure 2). When those modifications were written with $\Delta x=200 \mathrm{~nm}$ separation, cracks formed during the laser-writing (Figure 3); however, for larger separations the gratings were stable. The writing depth was approximately $10 \mu \mathrm{m}$ below the surface and extending into the sample. Strong stress-induced birefringence was observed inside the gratings in the regions without laser damage as well as between the gratings as revealed by cross-polarized imaging.

To determine the sign of refractive index change $\Delta n=n_{e}-n_{0}$, a $\lambda$-waveplate at $530 \mathrm{~nm}$ was inserted at $45^{\circ}$ in respect to the orientation of polarizer and analyzer. In this setting, Michael-Levy color charts can be used to determine color changes corresponding to $+|\Delta n|$ and $-|\Delta n|$ (Figure 3c). For the $\lambda$-plate of $530 \mathrm{~nm}$ wavelength oriented vertically, the blue color indicates the stress-induced regions. Since the blue color on the Michael-Levy chart corresponds to the higher absolute birefringence and the orange to the lower, the change $\Delta n$ has to be negative $n_{e}>n_{0}$ where $n_{0}$ is refractive index of the ordinary beam (perpendicular to the optical axis $O A$ ). The slow axis of the form-birefringent pattern (grating) is along the vertical direction (Figure 3c) and the refractive index is $\Delta n=n_{\|}-n_{\perp}=$ $n_{e}-n_{o}>0$. Hence, the form-birefringent structure acts as a negative uniaxial crystal.

To determine birefringence $\Delta n(\lambda)$ at several wavelengths, a recently developed method was used for measuring [33]. A single wavelength measurement of birefringence (as for example in the popular Abrio tool) leaves an ambiguity of the true $\Delta n$ value due to a possible $2 \pi$ folding of the phase and is 
avoided by carrying out measurements at several wavelengths in our method [33]. The measurement of $\Delta n$ was made at five different wavelengths while using narrow 10-nm-bandpass filters spanning the visible spectral range of $475-650 \mathrm{~nm}$. A linear fit through the origin point in the retardance $(\Delta n \times d[\mathrm{~nm}])$ vs. wavenumber $(1 / \lambda)$ plot was obtained with a single slope which defines the $\Delta n$ averaged over the tested spectral range [33]. Figure 4 shows the experimentally determined retardance for the grating patterns. Regions of interest (ROIs) were set to average retardance on the grating and in its vicinity. The error bars are the max and min retardance measured in the ROIs. The highest $\Delta n \times d$ was observed in between gratings and was scaling with separation $\Delta x$ between nano-planes. For the largest retardance of $112 \mathrm{~nm}$ and stretch parameter of $11(d=40 \mu \mathrm{m}), \Delta n=2.8 \times 10^{-3}$. Only slightly smaller birefringence was determined for the stretch factor of 10 and $30-\mu \mathrm{m}$-long inscribed gratings (Figure 4).

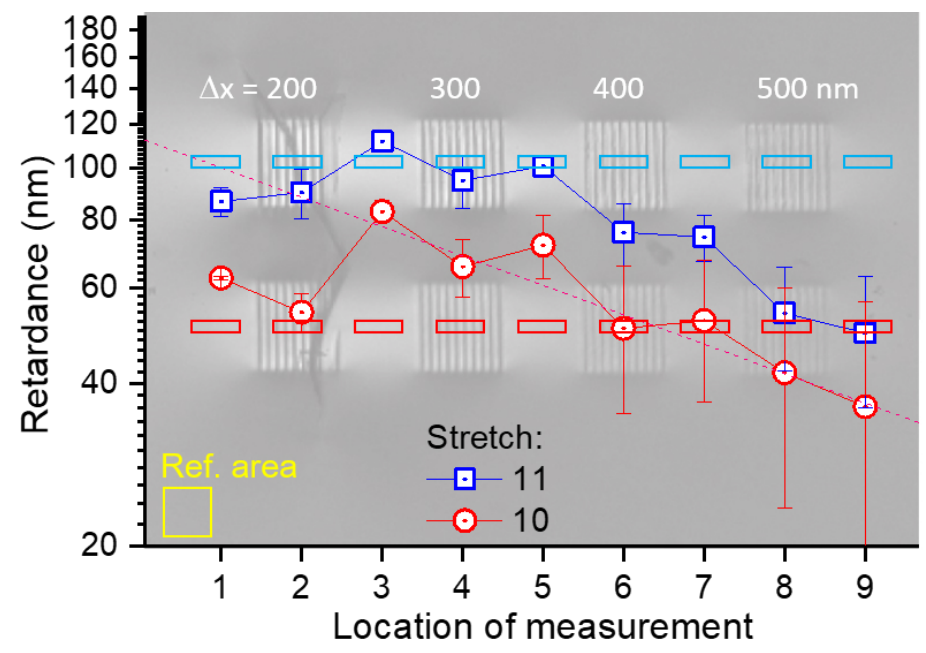

Figure 4. Retardance $|\Delta n| d$ measured at several wavelengths from $475 \mathrm{~nm}$ to $650 \mathrm{~nm}$ with 10-nm-bandpass filters. Sample was fs-laser-inscribed at pulse energy $E_{p}=574 \mathrm{~nJ}$; sample is shown in Figure 3. Rectangular regions of interest (ROIs) show locations from where an average retardance was measured. Two lines of gratings with different stretch factors of 11 (the length of inscribed line $d=40 \mu \mathrm{m})$ and $10(d=30 \mu \mathrm{m})$ were analyzed using liquid crystal compensator [33]. Note logarithmic ordinate was used to reveal single exponential decay of retardance with $\Delta x$.

Even the larger $\Delta n$ values were observed inside gratings in the $10-\mu \mathrm{m}$-wide openings reaching $22 \%$ retardance at the longest wavelength of $650 \mathrm{~nm}$, selected for the measurement of birefringence (Figure 5a). For shorter wavelengths in visible range, a $\lambda / 4$ waveplate condition was achieved by direct write of nano-inscribed modifications without changing the axial position of the modified region during inscription. Single-pixel cross-sections (Figure $5 b$ ) of the retardance maps show difference in the gradient of $\Delta n$ between laser-inscribed regions. By placing a few regions of nano-planes at different depths from the surface, it should be possible to fabricate $\lambda / 2$ waveplate retarders. Stress-induced regions between larger extended laser-structured patterns should allow reduction of light scattering observed from laser-inscribed areas which are used for fabrication of optical elements $[4,34]$.

Optical resolution of large area birefringence mapping (Figure 5) have provided only a couple of points measured per $10 \mu \mathrm{m}$ regions inside the grating. It illustrates a $0.1 \lambda$ modulation of retardance. Measurements at approximately twice a higher resolution (Figure 6) confirmed the modulation amplitude of retardance shown in Figure 5. Retardance at $625 \mathrm{~nm}$ wavelength (Figure 6) is shown in relative units of waves since the reference retardance required to make calibration was not possible to measure from the stress-free region on the same image (as area "Ref." in Figure 3). The cross sections of the measured retardance map clearly shows that stress-induced phase delay between inscribed $20-\mu \mathrm{m}$ period and 0.5 duty cycle grating were clearly resolved between the laser-inscribed regions with axial extension tens-of- $\mu \mathrm{m}$. 
(a)

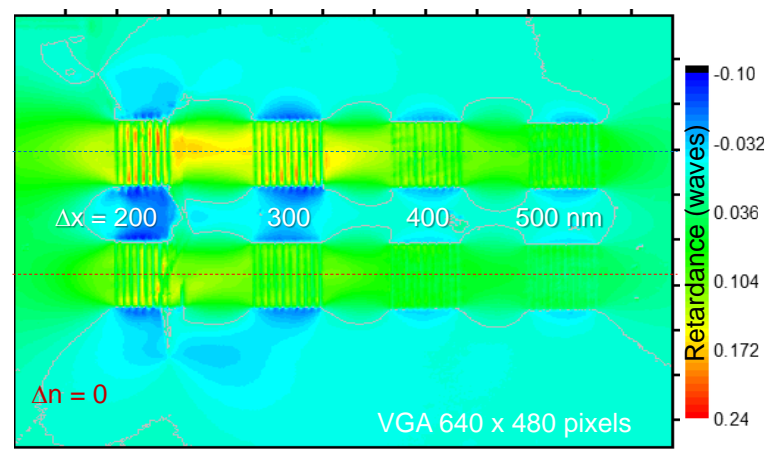

(b)

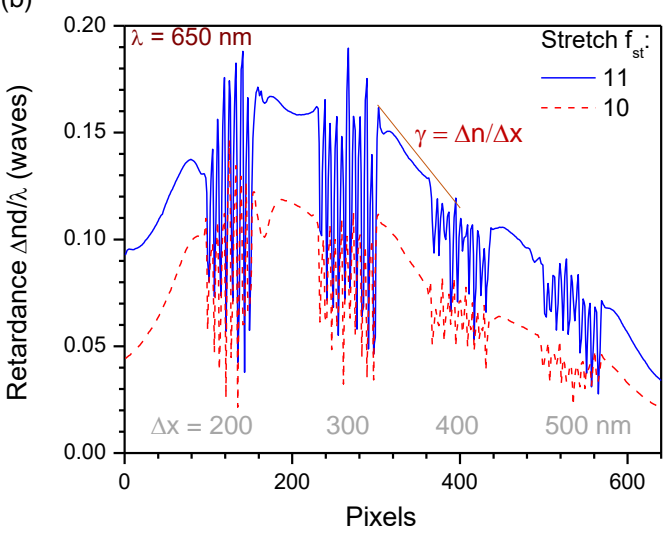

Figure 5. Retardance $|\Delta n| d / \lambda$ [waves] measured at $650 \mathrm{~nm}$; sample are shown in Figure 3 . (a) Retardance map calculated at a single pixel level for VGA $640 \times 480$ pixel area. The $\delta n=0$ contour lines are shown to distinguish regions affected by stress-induced birefringence; the maximum was 0.22 . Horizontal single-pixel cross sections are plotted in (b). The slope of retardance $\gamma=4.8 \times 10^{-4} /$ pixel or $\left(3.27 \times 10^{-4}\right) / \mu \mathrm{m}$ at the used magnification was achieved. One pixel corresponds to $1.4 \mu \mathrm{m}$ in the image while the optical resolution for the $N A=0.2$ lens was $0.61 \lambda / N A=2 \mu \mathrm{m}$.
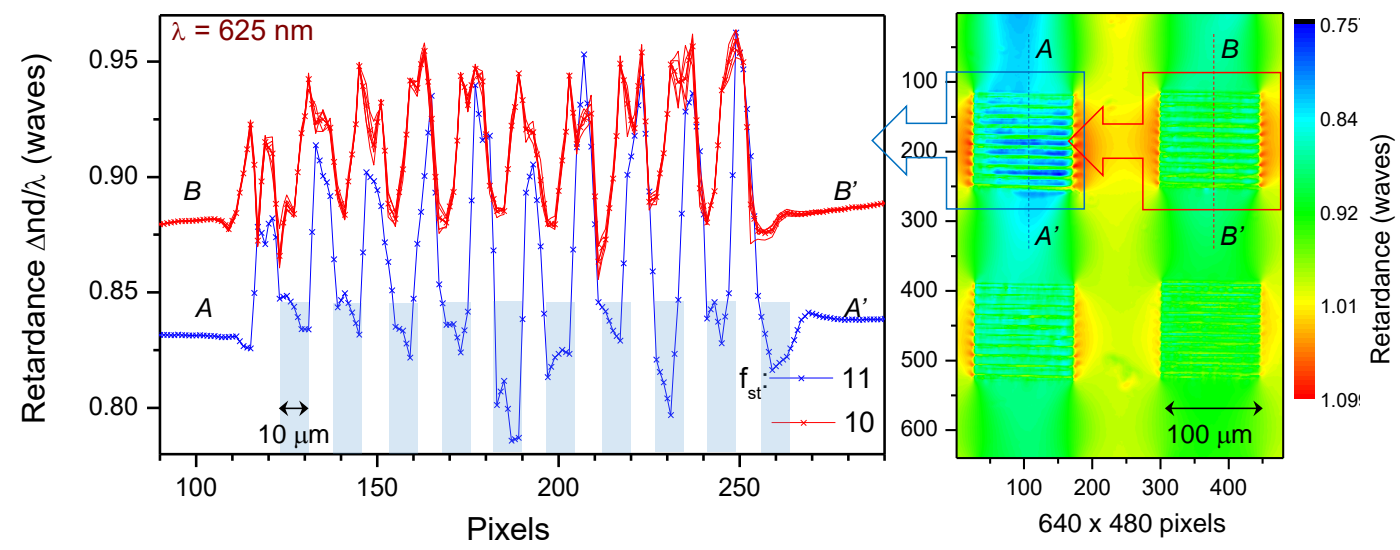

Figure 6. Retardance $|\Delta n| d / \lambda$ [waves] measured at $625 \mathrm{~nm}$ with higher resolution $N A=0.4$; sample are shown in Figures 3 and 5. Retardance map calculated at a single pixel level. Cross sections for two regions inscribed with stretch factors $f_{s t}=11$ and 10 are shown as one-pixel line for $\Delta x=200 \mathrm{~nm}$; for $f_{s t}=10$ five separate lines and their average $(\times$ marker in red line $)$ are plotted. The optical resolution for the $N A=0.4$ lens was $0.61 \lambda / N A=0.95 \mu \mathrm{m}$. Rectangular markers show positions of the inscribed regions for the $f_{s t}=11$ grating.

\section{Conclusions and Outlook}

We demonstrate $\lambda / 4$ phase retardance at visible wavelengths in sapphire recorded by direct write of nano-inscribed modifications tens-of-micrometers long. This modality of laser structuring opens a flexibility in stress-induced optical element fabrication and eliminates light scattering since the regions of tailored birefringence are outside of laser-structured regions. Patterns of tailored birefringence can be produced at different depths along the light propagation direction or even in different micro-plates for the final optical element. One particular field of application can be spin-orbital couplers where spatially variant birefringence can be inscribed with complex 3D topology similar to the polymerized 3D couplers [35]. 
Author Contributions: Conceptualization, S.J.; Funding acquisition, M.R., J.M. (Junko Morikawa), S.J. and Q.-D.C.; Methodology, H.F., M.R., R.H., Z.-Z.L., L.W. and J.M. (Jovan Maksimovic); Project administration, S.J., Q.-D.C. and H.-B.S.; Supervision, S.J., Q.-D.C. and H.-B.S.; Writing-original draft, H.F., R.H. and S.J.; Writing-review \& editing, H.F., M.R., J.M. (Junko Morikawa), Z.-Z.L., L.W., J.M. (Jovan Maksimovic) and S.J.

Funding: Partial funding is acknowledged: S.J. via the ARC Discovery DP190103284 and DP170100131 grants, J.M. (Junko Morikawa) by JSPS KAKENHI Grant No. 18H04506, Japan, M.R. by JSPS KAKENHI Grant No. 18J14350, Japan. H.F. by Graduate Interdisciplinary Research Fund of Jilin University No. 10183201844. H.F.,Z.-Z.Li, L.W., Q.-D.C and H.-B.S by the National Key Research and Development Program of China and National Natural Science Foundation of China (NSFC) under Grants \#2017YFB1104600, \#61825502, \#61590930, \#61827826, and \#61435005.

Acknowledgments: S.J. is grateful for support via the ChangJiang Distinguished Professor project on 3D laser nano/micro-printing at Jilin University.

Conflicts of Interest: The authors declare no conflicts of interest

\section{References}

1. Davis, K.M.; Miura, K.; Sugimoto, N.; Hirao, K. Writing waveguides in glass with a femtosecond laser. Opt. Lett. 1996, 21, 1729-1731. [CrossRef]

2. Bellouard, Y.; Said, A.; Dugan, M.; Bado, P. Fabrication of high-aspect ratio, micro-fluidic channels and tunnels using femtosecond laser pulses and chemical etching. Opt. Express 2004, 12, 2120-2129. [CrossRef]

3. Osellame, R.; Hoekstra, H.; Cerullo, G.; Pollnau, M. Femtosecond laser microstructuring: An enabling tool for optofluidic lab-on-chips. Laser Photonics Rev. 2011, 5, 442-463. [CrossRef]

4. Beresna, M.; Gecevicius, M.; Kazansky, P.; Gertus, T. Radially polarized optical vortex converter created by femtosecond laser nanostructuring of glass. Appl. Phys. Lett. 2011, 98, 201101. [CrossRef]

5. Bhuyan, M.K.; Courvoisier, F.; Lacourt, P.A.; Jacquot, M.; Salut, R.; Furfaro, L.; Dudley, J.M. High aspect ratio nanochannel machining using single shot femtosecond Bessel beams. Appl. Phys. Lett. 2010, 97, 081102. [CrossRef]

6. Wang, G.; Yu, Y.; Jiang, L.; Li, X.; Xie, Q.; Lu, Y. Cylindrical shockwave-induced compression mechanism in femtosecond laser Bessel pulse micro-drilling of PMMA. Appl. Phys. Lett. 2017, 110, 161907. [CrossRef]

7. Duocastella, M.; Arnold, C.B. Bessel and annular beams for materials processing. Laser Photonics Rev. 2012, 6, 607. [CrossRef]

8. Stoian, R.; Bhuyan, M.; Rudenko, A.; Colombier, J.P.; Cheng, G. High-resolution material structuring using ultrafast laser non-diffractive beams. Adv. Phys. X 2019, in press. [CrossRef]

9. Han, C.; Lee, M.; Callard, S.; Seassal, C.; Jeon, H. Lasing at topological edge states in a photonic crystal L3 nanocavity dimer array. Light Sci. Appl. 2019, 8, 40. [CrossRef]

10. Zhang, Q.; Yu, H.; Barbiero, M.; Wang, B.; Gu, M. Artificial neural networks enabled by nanophotonics. Light Sci. Appl. 2019, 8, 42. [CrossRef]

11. Augenstein, Y.; Vetter, A.; Lahijani, B.V.; Herzig, H.P.; Rockstuhl, C.; Kim, M.S. Inverse photonic design of functional elements that focus Bloch surface waves. Light Sci. Appl. 2018, 7, 104. [CrossRef]

12. Juodkazis, S.; Nishimura, K.; Misawa, H.; Ebisui, T.; Waki, R.; Matsuo, S.; Okada, T. Control over the State of Crystallinity: Sapphire. Adv. Mat. 2006, 18, 1361-1364. [CrossRef]

13. Liu, X.Q.; Chen, Q.D.; Guan, K.M.; Ma, Z.C.; Yu, Y.H.; Li, Q.K.; Tian, Z.N.; Sun, H.B. Dry-etching-assisted femtosecond laser machining. Laser Photonics Rev. 2017, 11, 1600115. [CrossRef]

14. Wei, D.; Wang, C.; Wang, H.; Hu, X.; Wei, D.; Fang, X.; Zhang, Y.; Wu, D.; Hu, Y.; Li, J.; et al. Experimental demonstration of a three-dimensional lithium niobate nonlinear photonic crystal. Nat. Photonics 2018, 12, 596. [CrossRef]

15. Ròdenas, A.; Gu, M.; Corrielli, G.; Paié, P.; John, S.; Kar, A.; Osellame, R. Three-dimensional femtosecond laser nanolithography of crystals. Nat. Photonics 2019, 13, 105-109. [CrossRef]

16. Sun, H.; Xu, Y.; Juodkazis, S.; Sun, K.; Watanabe, M.; Matsuo, S.; Misawa, H.; Nishii, J. Arbitrary-Lattice photonic Crystals Created by Multiphoton Microfabrication. Opt. Lett. 2001, 26, 325-327. [CrossRef]

17. Salter, P.; Baum, M.; Alexeev, I.; Schmidt, M.; Booth, M. Exploring the depth range for three-dimensional laser machining with aberration correction. Opt. Express 2014, 22, 17644-17656. [CrossRef] 
18. Rapp, L.; Meyer, R.; Giust, R.; Furfaro, L.; Jacquot, M.; Lacourt, P.A.; Dudley, J.M.; Courvoisier, F. High aspect ratio micro-explosions in the bulk of sapphire generated by femtosecond Bessel beams. Sci. Rep. 2016, 6, 34286. [CrossRef]

19. Fernandes, L.A.; Grenier, J.R.; Herman, P.R.; Aitchison, J.S.; Marques, P.V. Stress induced birefringence tuning in femtosecond laser fabricated waveguides in fused silica. Opt. Express 2012, 20, 24103-24114. [CrossRef]

20. Yang, S.M.; Hong, S.; Kim, S.Y. Optical, mechanical, and photoelastic anisotropy of biaxially stretched polyethylene terephthalate films studied using transmission ellipsometer equipped with strain camera and stress gauge. J. Polym. Sci. Part B Polym. Phys. 2019, 57, 152-160. [CrossRef]

21. Born, M.; Wolf, E. Principles of Optics: Electromagnetic Theory of Propagation, Interference and Diffraction of Light; Elsevier: Amsterdam, The Netherlands, 2013.

22. Morikawa, J.; Orie, A.; Hashimoto, T.; Juodkazis, S. Thermal and optical properties of the femtosecond-laser-structured and stress-induced birefringent regions of sapphire. Opt. Express 2010, 18, 8300-8310. [CrossRef]

23. Kazansky, P.; Inouye, H.; Mitsuyu, T.; Miura, K.; Qiu, J.; Hirao, K.; Starrost, F. Anomalous anisotropic light scattering in Ge-doped silica glass. Phys. Rev. Lett. 1999, 82, 2199-2202. [CrossRef]

24. Marcinkevicius, A.; Juodkazis, S.; Watanabe, M.; Miwa, M.; Matsuo, S.; Misawa, H.; Nishii, J. Femtosecond Laser-Assisted Three-Dimensional Microfabrication in Silica. Opt. Lett. 2001, 26, 277-279. [CrossRef]

25. Juodkazis, S.; Nishimura, K.; Misawa, H. Three-dimensional laser structuring of materials at tight focusing. Chin. Opt. Lett. 2007, 5, S198-S200.

26. Kudrius, T.; Šlekys, G.; Juodkazis, S. Surface-texturing of sapphire by femtosecond laser pulses for photonic applications. J. Phys. D Appl. Phys. 2010, 43, 145501. [CrossRef]

27. Juodkazis, S.; Matsuo, S.; Misawa, H.; Mizeikis, V.; Marcinkevicius, A.; Sun, H.B.; Tokuda, Y.; Takahashi, M.; Yoko, T.; Nishii, J. Application of femtosecond laser pulses for microfabrication of transparent media. Appl. Surf. Sci. 2002, 197-198, 705-709. [CrossRef]

28. Juodkazis, S.; Yamasaki, K.; Mizeikis, V.; Matsuo, S.; Misawa, H. Formation of Embedded Patterns in Glasses Using Femtosecond Irradiation. Appl. Phys. A 2004, 79, 1549-1553. [CrossRef]

29. Wang, L.; Xu, B.B.; Cao, X.W.; Li, Q.K.; Tian, W.J.; Chen, Q.D.; Juodkazis, S.; Sun, H.B. Competition between subwavelength and deep-subwavelength structures ablated by ultrashort laser pulses. Optica 2017, 4, 637-642. [CrossRef]

30. Gamaly, E.; Juodkazis, S.; Rode, A. Extreme Energy Density Confined Inside a Transparent Crystal: Status and Perspectives of Solid-Plasma-Solid Transformations. Nanomaterials 2018, 8, 555. [CrossRef]

31. Dharmavarapu, R.; Bhattacharya, S.; Juodkazis, S. Diffractive optics for axial intensity shaping of Bessel beams. J. Opt. 2018, 20, 085606. [CrossRef]

32. Juodkazis, S.; Nishimura, K.; Tanaka, S.; Misawa, H.; Gamaly, E.E.; Luther-Davies, B.; Hallo, L.; Nicolai, P.; Tikhonchuk, V. Laser-Induced Microexplosion Confined in the Bulk of a Sapphire Crystal: Evidence of Multimegabar Pressures. Phys. Rev. Lett. 2006, 96, 166101. [CrossRef]

33. Honda, R.; Ryu, M.; Li, J.L.; Mizeikis, V.; Juodkazis, S.; Morikawa, J. Simple multi-wavelength imaging of birefringence:case study of silk. Sci. Rep. 2018, 8, 17652. [CrossRef]

34. Beresna, M.; Brambilla, G.; Juodkazis, S.; Wang, X.; Rumpf, R. Geometric phase via stress induced birefringence. In Proceedings of the CLEO-Pacific Rim, Singapore, 31 July-4 August 2017; CLEO-PR Abstracts, p. 2.

35. Sanchez-Padilla, B.; Žukauskas, A.; Aleksanyan, A.; Balčytis, A.; Malinauskas, M.; Juodkazis, S.; Brasselet, E. Wrinkled axicons: Shaping light from cusps. Opt. Express 2016, 24, 24075-24082. [CrossRef]

(C) 2019 by the authors. Licensee MDPI, Basel, Switzerland. This article is an open access article distributed under the terms and conditions of the Creative Commons Attribution (CC BY) license (http:/ / creativecommons.org/licenses/by/4.0/). 